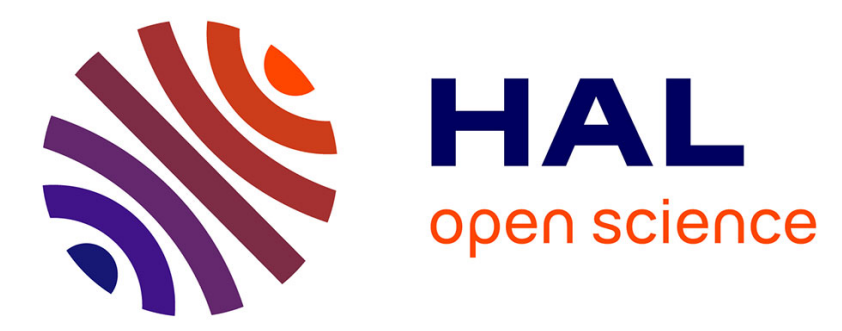

\title{
Elaboration du rendement et fertilisation azotée du blé d'hiver en Champagne crayeuse II. - Types de réponse à la fumure azotée et application de la méthode du bilan prévisionnel
}

\author{
Jean-Marc Meynard, Jean Boiffin, Jacques Caneill, Michel Sebillotte
}

\section{To cite this version:}

Jean-Marc Meynard, Jean Boiffin, Jacques Caneill, Michel Sebillotte. Elaboration du rendement et fertilisation azotée du blé d'hiver en Champagne crayeuse II. - Types de réponse à la fumure azotée et application de la méthode du bilan prévisionnel. Agronomie, 1981, 1 (9), pp.795-806. hal-00884326

\section{HAL Id: hal-00884326 \\ https://hal.science/hal-00884326}

Submitted on 1 Jan 1981

HAL is a multi-disciplinary open access archive for the deposit and dissemination of scientific research documents, whether they are published or not. The documents may come from teaching and research institutions in France or abroad, or from public or private research centers.
L'archive ouverte pluridisciplinaire HAL, est destinée au dépôt et à la diffusion de documents scientifiques de niveau recherche, publiés ou non, émanant des établissements d'enseignement et de recherche français ou étrangers, des laboratoires publics ou privés. 


\section{Elaboration du rendement et fertilisation azotée du blé d'hiver en Champagne crayeuse}

\section{II. - Types de réponse à la fumure azotée et application de la méthode du bilan prévisionnel}

Jean-Marc MEYNARD, Jean BOIFFIN, Jacques CANEILL, Michel SEBILLOTTE

I.N.R.A., Laboratoire de Recherches de la Chaire d'Agronomie, Institut National Agronomique ParisGrignon, 16, rue Claude-Bernard, F 75231 Paris Cedex 05.

\section{RÉSUMÉ}

Blé d'hiver,

Fertilisation azotée, Prévision des fumures, Courbes de réponse, Facteur limitant,

Etat structural du profil, Absorption d'azote, Objectif de rendement.
On a cherché à tester, en Champagne crayeuse, la méthode de détermination des fumures azotées du blé d'hiver d'après un bilan prévisionnel de l'azote minéral. Lors de 3 campagnes d'expérimentation, 120 parcelles expérimentales ont été implantées chez des agriculteurs.

Dans une première approche, on constate que la méthode n'a pas toujours permis d'obtenir les rendements pris comme objectifs (fig. 1 ). Mais l'utilisation du nombre de grains par $\mathrm{m}^{2}$ comme critère de jugement de l'efficacité de l'azote permet d'analyser les cas où la méthode semble en défaut ; on distingue alors 4 types de courbes de réponse (tabl. 5 et fig. 3 ):

- Type $\alpha$ : La dose calculée permet d'atteindre le nombre de grains-objectif.

- Type $\beta$ : Le nombre de grains $/ \mathrm{m}^{2}$ plafonne à un niveau très inférieur à l'objectif. On met alors en cause l'intervention précoce d'un facteur limitant la croissance, tel que parasitisme fongique, carence potassique, déficit hydrique (tabl. 7). L'erreur de prévision n'est pas à imputer à la méthode de calcul des fumures, mais seulement à un mauvais choix de l'objectif.

- Type $\gamma$ : Le nombre de grains $/ \mathbf{m}^{2}$ obtenu avec la dose d'engrais calculée est inférieur à l'objectif, alors que l'azote est encore facteur limitant. On invoque une mauvaise absorption de l'engrais par la culture, liée aux états structuraux défavorables constatés dans ces situations (tabl. 7). La méthode du bilan prévisionnel, qui suppose une absorption optimale de l'azote apporté par l'engrais, est alors en défaut.

- Type $\delta$ : L'objectif de nombre de grains $/ \mathrm{m}^{2}$ est atteint pour une dose de fumure inférieure à la dose calculée.

On conclut à la valeur de la méthode testée, qui a permis pour 80 p. 100 des parcelles où l'objectif de rendement n'était pas surestimé et où l'état structural était favorable, d'obtenir au moins le rendement prévu à la dose prévue.

\section{SUMMARY}

Winter wheat, Nitrogen fertilization, Prevision of fertilizing rates,

Response curves, Limiting factor, Soil structure,

Nitrogen absorption, Yield objective.
Yield elaboration processes and nitrogen fertilization of winter wheat in Champagne crayeuse. II Types of response to nitrogen fertilization and use of the predictive balance method

In Champagne crayeuse of France, we tried to test the determination method of nitrogen fertilizer rates on winter wheat, based on a predictive nitrogen balance. During 3 years campaigns 120 experimental and survey plots were settled and controlled within agricultural plots.

In a first approach, we note that the observed yields were often different from the objectives (fig. 1). However, using grain number per unit area as a criterion of nitrogen efficiency, allowed us to analyse the cases of bad prevision. 4 types of response curves to nitrogen are distinguished (tabl. 5 and fig. 3 ):

- Type $\alpha$ : The calculated rate allows to obtain the objective of grain number.

- Type $\beta$ : The obtained grain number is limited lower than the objective. We assume the influence of a limiting factor at an early stage of growth : fungic diseases, potassium or water deficiencies (table 7). The error of the prevision consists in a bad choice of the objective, and the method is not gain-said.

- Type $\gamma$ : The grain number obtained with the calculated rate of fertilizer is lower than the objective while nitrogen is still a limiting factor. The bad soil structure observed on this plots is likely to have disturbed the absorption of the fertilizer (table 7). The « Method of prevision balance», which supposes an optimum absorption of the fertilizer nitrogen, is then caught at fault.

- Type $\delta$ : The objective grain number is reached with a fertilizer rate lower than the calculated one. We conclude in favour of the validity of the tested method, which allowed to obtain, in 8 cases out of 10 , when the yield objective was not over-estimated and the soil structure favourable, at least the previous yield at the previous fertilizer rate. 


\section{INTRODUCTION}

La mise au point des recommandations en matière de fertilisation azotée des céréales s'est heurtée depuis longtemps à l'extrême variabilité des courbes de réponse du rendement des cultures à la fumure. En France, l'intense effort d'expérimentation, mené pendant près de 10 ans sous l'égide du Syndicat Professionnel de l'Industrie des Engrais Azotés (S.P.I.E.A.), n'a pas permis d'aboutir à un système de conseils au niveau de la parcelle suffisamment précis. La variabilité des courbes de réponse renvoie à celle des multiples conditions de milieu qui interviennent tant sur les besoins en azote des peuplements végétaux que sur le cycle de l'azote dans le sol.

En corollaire, l'application par les agriculteurs d'une région déterminée, même relativement homogène, de règles uniformes de fumure, donne de mauvais résultats. C'est ce que nous avons constaté en Champagne crayeuse, dans le cadre d'une étude sur l'irrégularité et la stagnation des rendements en blé d'hiver obtenus par un groupe d'agriculteurs pourtant de forte technicité (Le C.E.T.A. de Romilly-sur-Seine (Aube) avec lequel cette étude a été réalisée). Cette étude a été présentée dans le mémoire précédent de cette série (BOIFFIN et al., 1981).

La méthode de détermination des fumures d'après un bilan prévisionnel de l'azote minéral du sol (HÉBERT, 1969, 1975 ; RÉMY \& HÉBERT, 1977) se présente comme une tentative de résolution générale de ces problèmes. En effet, elle s'appuie sur une expression simplifiée des connaissances théoriques sur la dynamique de l'azote dans le sol et la plante et prend ainsi en compte les principaux paramètres susceptibles de modifier la courbe de réponse d'une culture aux apports d'engrais azotés. Cette méthode a donc suscité l'intérêt de tous ceux ayant à élaborer des prescriptions en matière de fumure azotée. Cependant son application doit être contrôlée régionalement.

Du bilan prévisionnel se déduit un modèle prédictif de réponse de la culture à l'azote qui suscite 2 types de démarches :

- On peut contrôler ce modèle en confrontant les prédictions qu'il entraîne à des résultats expérimentaux obtenus soit par essais - c'est ce qu'ont entrepris de faire, à l'échelle nationale depuis la campagne 1975, les organismes de développement, au premier rang desquels l'Institut Technique des Céréales et Fourrages qui a soutenu le travail présenté ci-dessous - soit par enquêtes. De la sorte, on soumet la méthode à l'épreuve de la variabilité du champ cultivé, qui affecte de multiples paramètres, inclus ou non dans le modèle. L'acceptation de la méthode dépend du degré de répétabilité de l'accord prévision-observation.

- On peut aussi utiliser ce modèle comme outil de description de la variabilité des courbes de réponse. En effet, celles-ci peuvent être situées en référence à la prévision et classées en fonction de la nature des écarts par rapport à celle-ci. Les cas de désaccord prévision-observation sont susceptibles d'une interprétation théorique, si l'on dispose d'observations complémentaires sur la situation culturale. Le modèle joue alors un rôle heuristique.

Le mémoire qui suit, expose comment on a tenté, dans le contexte précédemment cité, de mettre en œuvre cette double démarche d'utilisation d'un modèle théorique à des fins appliquées : proposition et contrôle de solutions techniques, d'une part ; inventaire et classification des différents types de réponse du blé aux engrais azotés, d'autre part ; on débouche en conclusion sur une discussion du modèle initial.

\section{CADRE DE L'ÉTUDE}

Le protocole retenu et les caractéristiques du milieu ont été présentés en détail dans un précédent mémoire (BoIFFIN et al., 1981). On en rappelle brièvement les grandes lignes.

- L'étude, pluriannuelle, a débuté en 1975. Seules les 3 premières campagnes sont analysées dans ce mémoire.

- Le dispositif associe enquête et expérimentation. L'enquête permet d'étudier le rôle du milieu et des pratiques culturales vis-à-vis de la croissance et l'élaboration du rendement ; les parcelles d'enquête sont appelées « parcelles types ». L'expérimentation sur les fumures azotées permet d'analyser l'influence de la nutrition azotée sur l'élaboration du rendement (essais).

Un réseau de parcelles d'un type intermédiaire relevant à la fois de l'enquête (par l'échantillonnage du milieu qu'il assure) et de l'expérimentation (3 doses d'azote sont mises en comparaison) a été adjoint au réseau initial de la $3^{\mathrm{e}}$ année d'étude (1977 : parcelles satellites). Le but de ces parcelles est de vérifier les possibilités d'extrapolation spatiale des résultats obtenus sur les 2 autres types de situations.

- A toutes les situations, on applique pour la fumure azotée la méthode du bilan prévisionnel proposée par HÉBERT (1969) :

La dose globale est prévue par l'équation :

$$
X=3 R+N_{\text {réc }}-N_{\text {hiv }}-N_{\text {rés }}-N_{\text {hum }}
$$

avec respectivement :

$\mathrm{R}$ : rendement objectif.

$\mathrm{N}_{\text {réc }}$ : reliquat minéral minimum après récolte.

$\mathrm{N}_{\text {hiv }}$ : reliquat d'azote minéral dans le sol en sortic d'hiver.

$\mathrm{N}_{\text {rés }}$ : azote minéralisé par les résidus organiques récents.

$\mathrm{N}_{\text {hum }}$ : azote minéralisé par l'humus du sol.

Le traitement résultant de l'application de la dose $\mathrm{X}$ et de la dose tallage $\mathrm{Y}$ est noté $(\mathrm{X}, \mathrm{Y})$.

- Pour toutes les situations, les modalités d'élaboration du rendement sont analysées, ce qui implique des contrôles nombreux permettant la compréhension du comportement de la végétation en relation avec les états du milieu. Sur les parcelles satellites, seuls les contrôles indispensables à la vérification des conclusions dont on teste l'extrapolation ont été retenus.

- Le choix des situations étudiées répond au souci de tester les prévisions découlant de l'application du modèle dans une gamme de situations faisant principalement varier les paramètres non pris en compte par le modèle, mais pourtant susceptibles de modifier la réponse du blé à la fumure azotée (à l'exception, bien entendu, du reliquat azoté en sortie d'hiver). En conséquence, on privilégie les variabilités liées au climat, au type de sol, à la technicité de l'agriculteur et aux aspects de l'histoire culturale ne concernant pas les flux d'azote. Au contraire, on se restreint autant que possible au seul précédent betterave et on évite la plupart du temps les défriches récentes (assez fréquentes dans cette région), pour homogénéiser les termes de minéralisation du bilan.

\section{RÉSULTATS ET DISCUSSION}

\section{A. Rendements et absorption d'azote}

\section{Présentation générale des rendements}

Les rendements obtenus sur les différentes parcelles du dispositif sont présentés sur le tableau 1. Ils sont en général 


\section{TABLEAU 1}

Présentation générale des rendements

General view of the obtained yields

Les rendements exprimés en q/ha de grain à 16 p. 100 d'humidité sont relatifs aux parcelles fertilisées aux doses $X, Y$ et traitées aux fongicides.

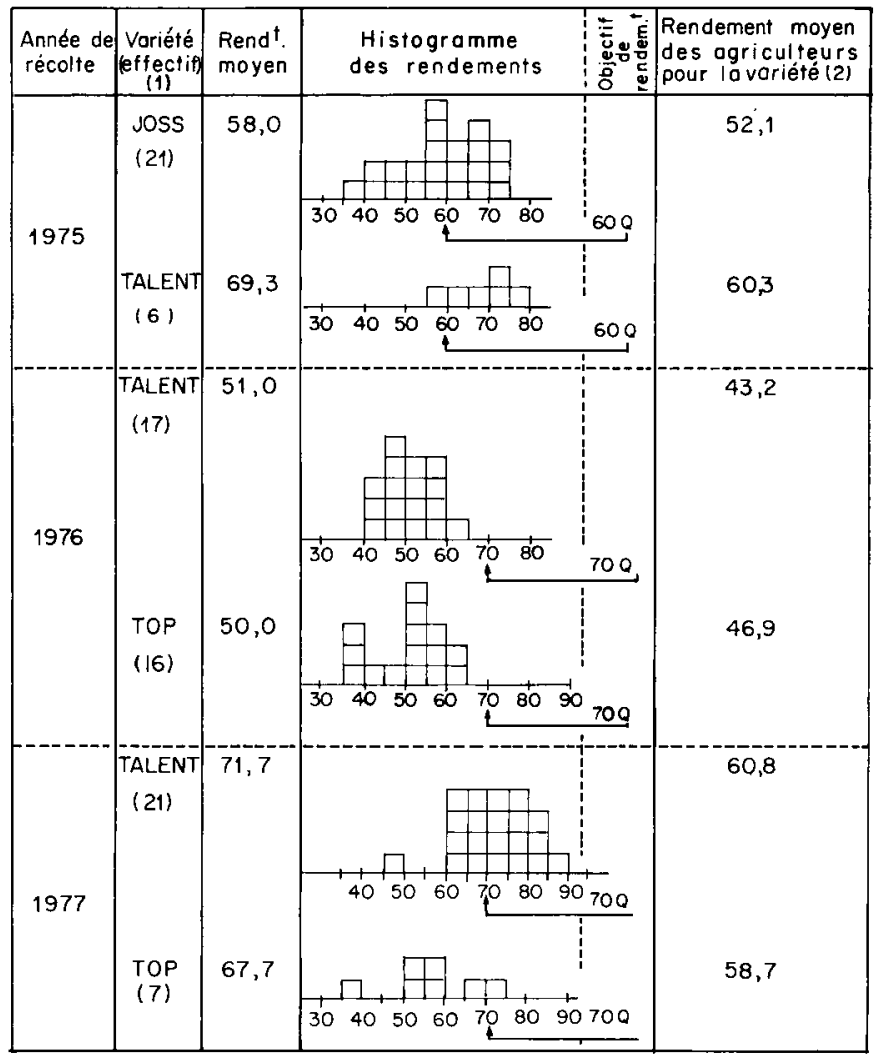

(') sont comptées les parcelles types et les parcelles correspondant au traitement de base sur les essais

${ }^{\left({ }^{2}\right)}$ d'après l'enquête annuelle du CETA de Romilly (F. FERraTON).

plus élevés que les rendements moyens des agriculteurs pour la variété considérée, ce qui est une première indication en faveur des méthodes de conduite préconisées. Cependant, l'objectif de rendement n'est pas toujours atteint et la variabilité entre parcelles reste importante : la méthode de prévision des fumures ne s'applique donc pas partout de façon indiscutable.
2. Effet des fumures azotées sur le rendement-Résultats des essais

L'effet de la dose globale d'engrais azoté sur le rendement apparaît sur la figure 1 (analyse statistique en annexe 1). Toutes années confondues, 3 grands types de réponse se distinguent :

Type $\alpha$ : L'objectif de rendement est atteint à la dose $X$ prévue

C'est le cas d'un essai (ECE) en 1975 et, à 3 q près, des essais EEB et EER en 1977. La prévision est bonne.

Type $\beta$ : Les rendements plafonnent à des niveaux très inférieurs à l'objectif de rendement

L'effet de la dose globale n'est pas significatif. C'est le cas de l'essai ENE en 1975 et des 4 essais en 1976. Une réponse de cette allure suggère l'influence d'un facteur limitant autre que l'azote. En 1976, on peut invoquer un double effet de la sécheresse, qui, d'une part a effectivement joué un rôle de facteur limitant direct, mais, d'autre part, a diminué l'efficacité des engrais, appliqués sur sol sec (SÉBILlotTE et al., 1978). En 1975, on a constaté en fin de végétation une très forte infestation parasitaire sur l'essai ENE (pucerons et rouille jaune principalement) qui a pu limiter la croissance en fin de cycle. Pour tous les cas, c'est la prévision de l'objectif de rendement qui est mise en défaut et non la méthode de prévision des fumures en elle-même.

Type $\gamma:$ Le rendement obtenu à la dose $X$ est inférieur à l'objectif, mais simultanément l'effet de la dose globale est significatif dans toute la gamme testée

L'azote est donc bien encore facteur limitant malgré les fortes fumures appliquées. La méthode de prévision est mise en défaut ; c'est le cas des essais ESO en 1975 et EON en 1977. On peut avancer 2 hypothèses principales :

- une fourniture d'azote par le milieu plus faible que prévu, - une mauvaise valorisation des engrais azotés (dont les courbes plates de 1976 peuvent représenter un cas limite, bien qu'on les ait classées de prime abord dans le type $\beta$ ).

\section{Bilans d'azote sur le traitement de base des essais}

La comparaison des bilans d'azote prévisionnels et réels - ces derniers étant établis en considérant que l'appareil racinaire représente 25 p. 100 de l'azote total contenu dans
Rendement en $q$ /ha
à 16 p. $100 d^{\prime}$ humidite

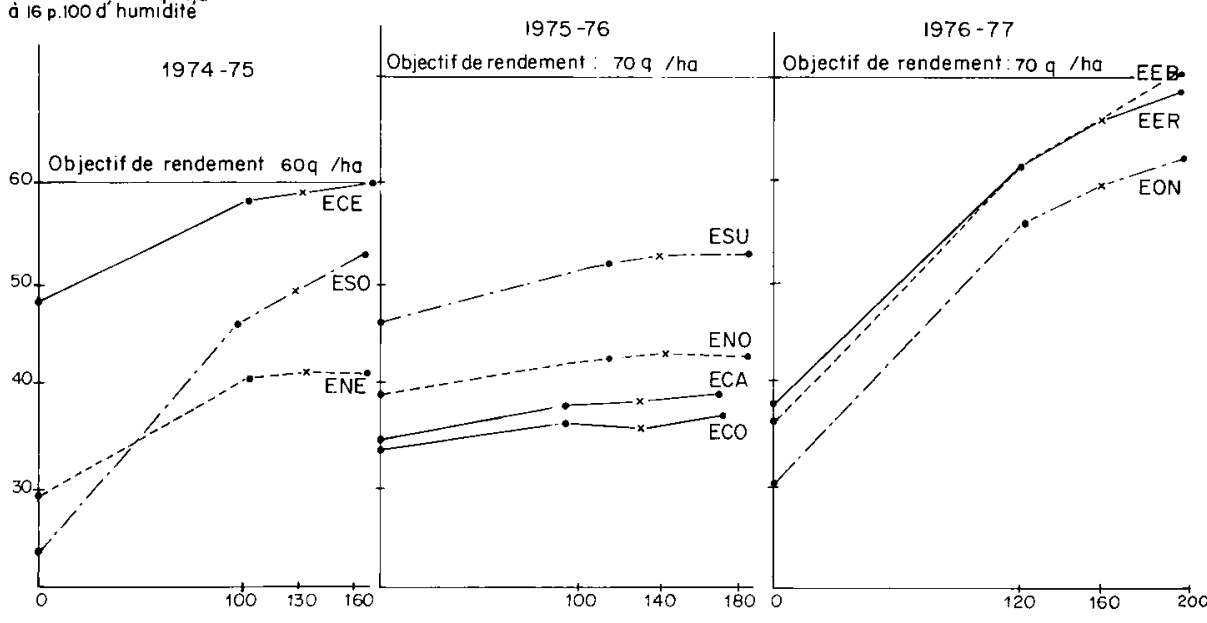

Figure 1

Effet de la dose globale d'azote apportée sur le rendement des essais (les points marqués « $X$ » correspondent aux traitements de base: dose totale $X$, dose tallage $Y$ ).

Response of wheat crops to nitrogen fertilization on the experimental plots (point noted $X$ correspond to the reference treatment total apply $X$, first apply $Y)$. 


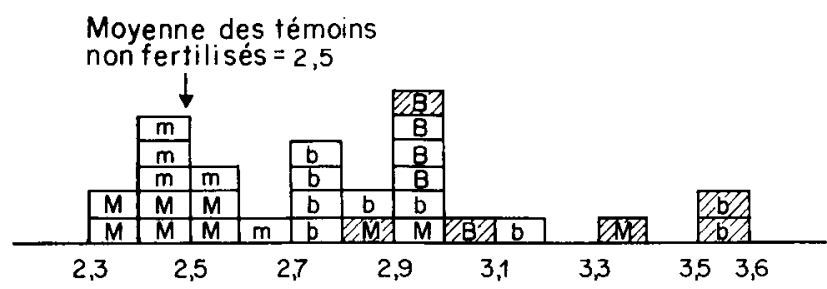

Figure 2

Quantité d'azote absorbé par quintal de grain produit sur les traitements de base des essais et parcelles types (1977)

Etat structural de la couche travaillée: $B=B o n ; b=$ moyen; $m=$ médiocre $; M=$ mauvais

Cases hachurées : situations parasitées ou carencées en potassium

$\mathrm{Kg} N$ absorbed for one $q$ grain yielded (experimental and survey plots; nitrogen rates $X, Y)$

Soil structure : $B=$ good ; $b=$ average ; $m=$ mediocre $; M=b a d$ Shaded parts: Situations with fungic diseases or potassium deficiencies

les parties aériennes - est un autre moyen de contrôle de la méthode de calcul de la dose $\mathrm{X}$. Cette comparaison est présentée dans le tableau 2.

L'absorption globale d'azote est le plus souvent inférieure à ce qui était prévu. Ceci est particulièrement net pour les essais où le rendement à la dose $\mathrm{X}$ n'atteint pas l'objectif (types de courbes $\beta$ et $\gamma$ ); ces essais se distinguent également par des reliquats d'azote nitrique après récolte particulièrement élevés. Dans le cas des courbes de la catégorie $\gamma$ (azote encore facteur limitant à la dose X), cette observation corrobore l'hypothèse d'une mauvaise valorisation des engrais azotés, d'autant plus que c'est surtout au niveau de la couche arable que les valeurs observées dépassent fortement les prévisions ( $10 \mathrm{~kg} \mathrm{~N}$ nitrique par tranche de $30 \mathrm{~cm}$ de sol). Or par ailleurs, on a observé (annexe 2), que les essais ESO en 1975, EON, EEB et EER en 1977, présentaient dans la couche arable un état structural défavorable avec de nombreuses mottes volumineuses et compactes non colonisées par les racines.

On peut donc penser que la colonisation faible et hétérogène du profil a perturbé l'assimilation des engrais azotés et de l'azote minéralisé. Cette influence de l'état structural est en accord avec les résultats obtenus sur d'autres cultures par BLANCHET et al. (1974), dans des conditions expérimentales très contrôlées, et par DRAY. COTT et al. (1970), au champ.

\section{Vue d'ensemble sur les rapports azote absorbé/rendements}

Pour les essais et parcelles types, ces rapports sont proches, en moyenne, de la norme de $3 \mathrm{~kg}$ d'azote $/ \mathrm{q}$ de grain, proposée par CoÏC en 1956, pour des blés bien alimentés : 2,92 en $1975 ; 2,91$ en $1976 ; 2,77$ en 1977.

TABLEAU 2

Bilans azotés sur les essais (d'après placettes)

(parcelles ayant reçu les doses $X, Y$ )

Nitrogen balances on the experimental plots

\begin{tabular}{|c|c|c|c|c|c|c|c|c|c|c|c|c|c|}
\hline \multirow{4}{*}{$\begin{array}{c}\text { POSTES DU } \\
\text { BILAN EN } \\
\mathrm{kg} \mathrm{N} / \mathrm{ha}\end{array}$} & \multicolumn{13}{|c|}{ ANNÉES ET ESSAIS } \\
\hline & \multirow{3}{*}{ Prévu } & \multicolumn{3}{|c|}{1975} & \multicolumn{5}{|c|}{1976} & \multicolumn{4}{|c|}{1977} \\
\hline & & \multicolumn{3}{|c|}{ valeurs obscrvées sur: } & \multirow{2}{*}{ Prévu } & \multicolumn{4}{|c|}{ valeurs observées sur: } & \multirow{2}{*}{ Prévu } & \multicolumn{3}{|c|}{ valeurs observées sur: } \\
\hline & & ENE & ECE & ESO & & ENO & ESU & ECA & $\mathrm{ECO}$ & & EON & EEB & EER \\
\hline azote absorbé & 180 & 145 & 209 & 93 & 210 & 114 & 145 & 121 & 113 & 210 & 174 & 166 & 196 \\
\hline $\begin{array}{l}\text { azote absorbé par quintal de } \\
\text { grain }(\mathrm{kg} \mathrm{N})\end{array}$ & 3 & 3.1 & 3.2 & 2.1 & 3 & 2.6 & 3.0 & 2.7 & 2.7 & 3 & 2.6 & 2.4 & 2.5 \\
\hline reliquat sortic d'hiver $\left({ }^{\prime}\right)$ & $\begin{array}{l}30 \\
33 \\
30\end{array}$ & 50 & 33 & 19 & $\begin{array}{l}46 \\
44 \\
53\end{array}$ & $>46$ & 35 & $>53$ & $>53$ & $\begin{array}{l}34 \\
36 \\
26\end{array}$ & 20 & 30 & 24 \\
\hline reliquat récolte $\left({ }^{1}\right)$ & 20 & 52 & 39 & 57 & 20 & $>50$ & 37 & $>77$ & $>110$ & 20 & 60 & 31 & 42 \\
\hline minéralisation nette $\left({ }^{2}\right)$ & 45 & 17 & 85 & 6 & 45 & cf. $\left({ }^{1}\right)$ & 7 & cf. $\left({ }^{1}\right)$ & cf. $\left({ }^{1}\right)$ & 45 & 54 & 7 & 44 \\
\hline $\begin{array}{l}\text { apport d'engrais azoté (dose } \\
\text { X) }\end{array}$ & $\begin{array}{l}125 \\
122 \\
125\end{array}$ & 130 & 130 & 125 & $\begin{array}{l}139 \\
141 \\
132\end{array}$ & 140 & 140 & 130 & 130 & $\begin{array}{l}151 \\
149 \\
159\end{array}$ & 160 & 160 & 160 \\
\hline $\begin{array}{l}\text { type de courbe de réponse du } \\
\text { Rendt. }\end{array}$ & & $\beta$ & $\alpha$ & $\gamma$ & & $\beta$ & $\beta$ & $\beta$ & $\beta$ & & $\gamma$ & $\alpha$ & $\alpha$ \\
\hline
\end{tabular}

(1) Colonne «Prévu»: valeurs mesurées ou estimées sur $60 \mathrm{~cm}$ (profondeur d'enracinement prévue).

Colonne «observé »: valcurs mesurées ou estimées sur la profondeur d'enracinement observée à la floraison, souvent différente de $60 \mathrm{~cm}$.

Le signe « $>$ indique que les profondeurs ayant été supérieures à $1 \mathrm{~m}$., le reliquat est supérieur à la valeur indiquée. La minéralisation n'a donc pu être calculée.

$\left({ }^{2}\right)$ Déduites du bilan pour la colonne «Valcurs observées ». 
Cependant la dispersion des valeurs n'est pas négligeable (de 2,31 à 3,60 en 1977). D'autre part, sur les essais, dans 7 cas sur 10 , elles sont nettement inférieures à la norme de $3 \mathrm{~kg} / \mathrm{q}$.

S'interrogeant sur la signification de ce critère vis-à-vis de la nutrition azotée, on peut examiner ses variations en fonction des doses d'azote appliquées sur les essais (il a paru inutile de présenter les résultats de 1976 , ceux-ci ne pouvant s'interpréter en terme de réponse à l'azote des engrais (SEBILlOTTE et al., 1978)). On observe un effet important et systématique des doses d'azote (tabl. 3). Des valeurs assez proches de la norme de $3 \mathrm{~kg}$ d'azote $/ \mathrm{q}$ sont en général atteintes pour des doses d'engrais correspondant au début du palier de la réponse à l'azote du rendement (chiffres en gras dans le tableau 3).

\section{TABLEAU 3}

Prélèvements d'azote sur les essais. Ramenés au quintal de grain récolté (à $16 \%$ d'humidité

Ratios $N$ absorbed/yield $\left(k g \cdot q^{-1}\right)$

\begin{tabular}{lcccc}
\hline & Témoin sans azote & $\mathrm{X}-\mathbf{3 0}$ & $\mathrm{X}$ & $\mathrm{X}+30$ \\
Essai ENE & 2,93 & $\mathbf{2 , 9 7}$ & 3,11 & 3,18 \\
Essai ECE & 2,72 & $\mathbf{2 , 9 9}$ & 3,19 & 3,32 \\
Essai ESO & 2,80 & 2,18 & 2,25 & 2,60 \\
& & & & \\
\hline & Témoin & $\mathrm{X}-40$ & $\mathrm{X}$ & $\mathrm{X}+40$ \\
Essai EON & 2,87 & 2,74 & 2,78 & $\mathbf{2 , 9 9}$ \\
Essai EEB & 2,31 & 2,40 & 2,46 & 2,62 \\
Essai EER & 2,25 & 2,46 & $\mathbf{2 , 5 2}$ & 2,80 \\
\hline \hline
\end{tabular}

En deçà de ces doses, l'absorption d'azote par quintal de grain est d'autant plus faible que la dose est plus faible, sauf pour certains témoins sans azote à rendement très bas ; cette diminution est conforme au schéma classique de dilution des éléments minéraux dans la matière sèche en conditions de très forte carence. Tous les essais pour lesquels on a pu invoquer, d'après l'observation du milieu, une perturbation de l'absorption d'azote (soit du fait de la sécheresse en 1976, soit du fait de l'état structural, pour ESO en 1975 et les 3 essais de 1977), présentent précisément à la dose $\mathrm{X}$ des valeurs inférieures à $3 \mathrm{~kg} / \mathrm{q}$ de grain, témoignant d'une mauvaise nutrition azotée.

Les résultats obtenus sur les parcelles types (fig. 2) permettent d'inventorier une gamme plus variée de milieux sur lesquels on dispose d'éléments de caractérisation, concernant :

- l'intervention de facteurs limitants graves. On a ainsi pu détecter des cas de carence en potasse ou de forte attaque parasitaire,

- l'état structural: les profils culturaux réalisés à la floraison ont fait l'objet d'une notation systématique portant sur la porosité et la cohésion de chaque horizon et sur la densité de l'enracinement. A partir de ces notations, on a formulé un jugement sur l'état structural de la couche travaillée, répartissant les profils en 4 classes ordonnées : état structural bon, moyen, médiocre ou mauvais. Les méthodes de description et de classement des profils sont exposées dans un précédent mémoire (Boiffin et al., 1981).

Les situations sur lesquelles un facteur limitant grave autre que l'azote et l'état structural a été identifié accusent des prélèvements par quintal de grain élevés (généralement supérieurs à $3 \mathrm{~kg}$ ). La nutrition azotée y est pléthorique.
A l'opposé, sur les situations dont l'état structural est mauvais ou médiocre, les prélèvements d'azote sont faibles, toujours inférieurs à $2,7 \mathrm{~kg} / \mathrm{q}$ en l'absence de facteur limitant mentionné ci-dessus et voisins de ceux que l'on peut constater sur les témoins non fertilisés. La nutrition azotée y est déficiente.

\section{Conclusion}

Le modèle linéaire qui traduit la méthode des bilans prévisionnels peut s'écrire en termes de prévision des rendements, soit :

$$
R=\frac{1}{a}\left(F+N_{\text {hiv }}+N_{\text {rés }}+N_{\text {hum }}-N_{\text {réc }}\right)
$$

Les symboles utilisés ont été explicités au § II (relation 1) ; F est la dose d'engrais appliquée et « $\mathbf{a} »$ est un coefficient qui traduit la quantité d'azote absorbé par quintal de grain formé. Au voisinage de l'optimum de réponse, « $\mathbf{a}$ » est voisin de $3 \mathrm{~kg}$ d'azote $/ \mathrm{q}$.

Son application parfaite dans le contexte étudié se traduirait par une homogénéisation des rendements obtenus à la dose $\mathrm{X}$, correspondant aux objectifs uniformes de $60 \mathrm{q}$ en $1975,70 \mathrm{q}$ en 1976 et 77 . Ce n'est pas le cas. Une importante variabilité des réponses à l'azote se manifeste avec 2 principaux cas de déviation par rapport à la prévision :

- réponse de type $\beta$, l'objectif de rendement est surestimé. Il n'y a alors pas lieu de chercher à vérifier la relation (2) dans la gamme de fumure testée ;

- réponse de type $\gamma$, l'azote est encore facteur limitant à la dose prévue, alors que les rendements sont inférieurs à l'objectif. La méthode est alors en défaut, dans son domaine de validité. Ces cas correspondent systématiquement à une nutrition azotée déficiente $(a<3)$.

On se propose de relier chacune de ces déviations à des conditions de milieu déterminées. Ainsi on a pu invoquer :

- l'intervention d'un facteur limitant autre que l'azote, dans le cas des courbes $\beta$;

- la perturbation de l'absorption d'azote, du fait d'un mauvais état structural du profil, dans le cas des courbes $\gamma$. L'application de la méthode du bilan prévisionnel repose, en effet, sur l'hypothèse implicite que l'ensemble de l'azote minéral du sol disponible depuis la sortie de l'hiver jusqu'à la récolte (engrais, reliquats, minéralisation) est utilisé par le blé.

Cependant les rendements obtenus et les quantités d'azote absorbées sont des critères très globaux qui intègrent toute l'histoire des relations plante-milieu.

Pour analyser cette histoire, on divisera le cycle du blé en 2 phases successives, correspondant à des processus bien différenciés et relativement indépendants :

- jusqu'à la fécondation, une phase de détermination du nombre de grains $/ \mathrm{m}^{2}$, qui correspond aussi à la mise en place de l'appareil foliaire et du système radiculaire ;

- une phase ultérieure plus courte de remplissage des grains formés, grâce à l'activité de l'appareil photosynthétique mis en place et sanctionnée par le poids de 1000 grains.

On a montré dans le mémoire précédent (BoIfFin et al., 1981) que l'on pouvait considérer le nombre de grains par unité de surface comme le critère le plus pertinent de la réponse à l'azote du blé, mieux approprié que le rendement, car ce dernier intègre la phase de remplissage des grains, qui fait intervenir des facteurs autres que la nutrition azotée. 
TABLEAU 4

Fixation des objectifs de nombre de grains par $m^{2}$ (les poids sont donnés à $15 \%$ d'humidité)

Choice of grain number objectives

\begin{tabular}{|c|c|c|c|c|}
\hline Variétés & $\begin{array}{c}\text { Poids de } 1000 \text { grains } \\
\text { moyen variétal }(\mathrm{g})\end{array}$ & $\begin{array}{l}\text { Campagne } \\
\text { d'étude }\end{array}$ & $\begin{array}{l}\text { Objectif de } \\
\text { rendement }(q / h a)\end{array}$ & $\begin{array}{l}\text { Objectif de nombre } \\
\text { de grains } / \mathrm{m}^{2}\end{array}$ \\
\hline JOSS & 42,85 & 1975 & 60 & 14000 \\
\hline \multirow[t]{2}{*}{ TALENT } & 38,10 & 1975 & 60 & 15750 \\
\hline & & $1976 \& 1977$ & 70 & 18400 \\
\hline TOP & 45,75 & $1976 \& 1977$ & 70 & 15300 \\
\hline
\end{tabular}

B. Le nombre de grains par unité de surface, critère de réponse à l'azote du peuplement du blé

Pour appliquer la méthode du bilan prévisionnel pour la fixation des fumures, il faut déterminer un objectif de rendement. Implicitement, celui-ci comporte un objectif de nombre de grains, et c'est ce dernier qu'il convient de mettre en relation avec la fertilisation azotée pour juger des conditions d'application de la méthode.

On doit déterminer ce nombre de grains $/ \mathrm{m}^{2}$-objectif, à la fois nécessaire et suffisant pour atteindre l'objectif de rendement dans des conditions de maturation de grain données. En supposant le parasitisme bien contrôlé et le déficit hydrique de début d'été modéré, on peut estimer, selon les variétés, les valeurs du poids moyen prévisible du grain ; elles figurent dans le tableau 4.

La méthode de prévision des fumures peut être considérée comme validée si le nombre de grains-objectif est atteint à la dose $\mathrm{X}$ et si toute dose inférieure donne un nombre de grains inférieur.

Par référence au schéma prévisionnel satisfaisant ces 2 conditions, on peut envisager les 3 types de réponse à la fertilisation azotée $(\alpha, \beta, \gamma)$, déjà définis à propos des rendements, et un $4^{\mathrm{e}}$ type $(\delta)$, pour lequel l'objectif est atteint à une dose de fumure inférieure à $\mathrm{X}$. Cette typologie consiste en fait à prendre en compte 2 critères :

- la différence entre le nombre de grains $/ \mathrm{m}^{2}$ atteint à la dose $\mathrm{X}$ et l'objectif ;

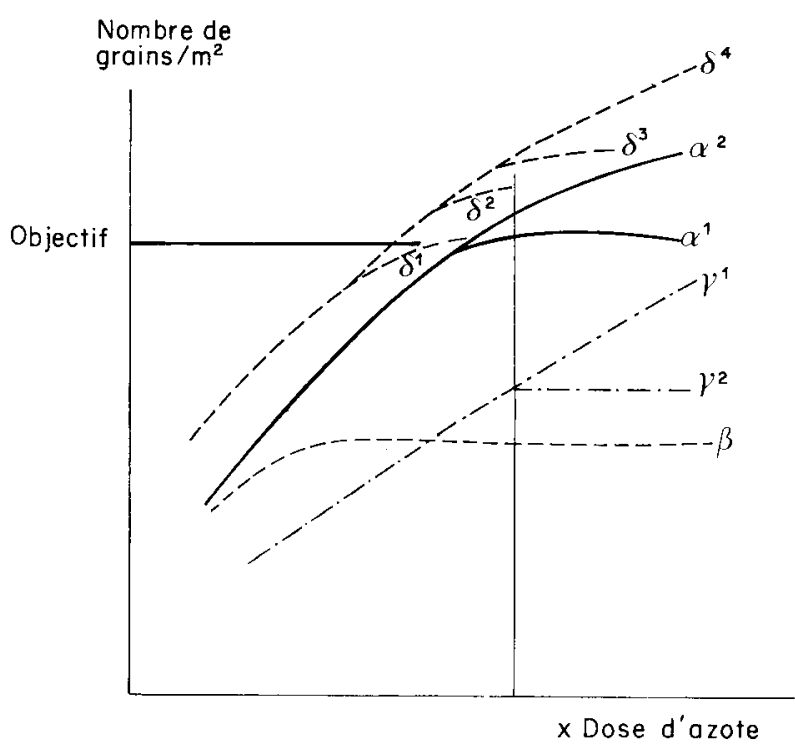

Figure 3

Types de courbes de réponse à l'azote

Types of response curves to nitrogen
- la dose d'azote minimum permettant d'atteindre le plus fort nombre de grains de l'essai considéré.

La correspondance entre ces critères et la typologie des courbes est présentée sur la figure 3 et dans le tableau 5.

TABLEAU 5

Types de courbes de réponse à l'azote

Types of response curves to nitrogen

\begin{tabular}{|c|c|c|c|c|}
\hline & & \multicolumn{3}{|c|}{$\begin{array}{l}\text { Dose d'azote la plus faible pour } \\
\text { laquelle on obtient le nombre de } \\
\text { grains maximum de l'essai }\end{array}$} \\
\hline & & $<\mathrm{x}$ & $\mathrm{x}$ & $>x$ \\
\hline \multirow{3}{*}{$\begin{array}{l}\text { Nombre de } \\
\text { grains } \\
\text { à la dose } x\end{array}$} & \multirow{3}{*}{$\begin{array}{r}>\text { Objectif } \\
\text { Objectif } \\
<\text { Objectif }\end{array}$} & $\delta_{2}$ & $\delta_{3}$ & $\delta_{4}$ \\
\hline & & $\delta_{1}$ & $\alpha_{1}$ & $\alpha_{2}$ \\
\hline & & $\beta$ & $\gamma_{2}$ & $\gamma_{1}$ \\
\hline
\end{tabular}

On peut alors confronter cette typologie avec les résultats obtenus sur les essais complets ou simplifiés (parcelles satellites de 1977): on obtient globalement la répartition indiquée dans le tableau 6.

TABLEAU 6

Proportion des différents types de courbes en 1977 (essais et parcelles satellites, 35 situations)

Proportion of the different curve types in 1977 (35 situations)

\begin{tabular}{cc}
\hline$\alpha_{1}+\alpha_{2}$ & $36 \%$ \\
\hline$\beta$ & $22 \%$ \\
\hline$\gamma_{1}+\gamma_{2}$ & $31 \%$ \\
\hline$\delta_{1}+\delta_{2}+\delta_{3}+\delta_{4}$ & $11 \%$ \\
\hline
\end{tabular}

Comme pour les parcelles types, on peut caractériser le milieu de chaque essai par des informations complémentaires. Elles concernent l'identification de facteurs limitants précoces, intervenus avant la floraison, (carences en potasse ou magnésie, parasitisme précoce, alimentation hydrique en 1976), et l'état structural de la couche travaillée, condition de la croissance. 
TABLEAU 7

Conditions de milieu et types de réponse à la fertilisation azotée (parcelles satellites et essais)

Ecological conditions and response curves to nitrogen fertilization

\begin{tabular}{|c|c|c|c|c|}
\hline & & \multicolumn{3}{|c|}{ Maximum de nombre de grains $/ \mathrm{m}^{2}$ atteint dès: $\left({ }^{1}\right)$} \\
\hline & & $x-40$ & $\mathrm{x}$ & $x+40$ \\
\hline \multirow{3}{*}{ 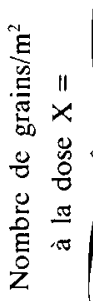 } & Objectif $+5 \grave{a}+15 \%$ & b & $\mathrm{B}, \mathrm{b}$ & \\
\hline & Objectif -5 à $+5 \%$ & $b_{75}, B$ & $\mathrm{~B}, \mathrm{~b}, \mathrm{~b}, \mathrm{~m}_{77}, \mathrm{M},(\mathrm{B}), \mathrm{b}_{75}$ & $\mathrm{~B}, \mathrm{~B}, \mathrm{~b}, \mathrm{M}, \mathrm{M}, \mathrm{M}$ \\
\hline & $\begin{array}{l}\text { Objectif }-15 \text { à }-5 \% \\
\text { Objectif }-25 \text { à }-15 \% \\
\text { Objectif }-25 \text { à }-45 \%\end{array}$ & $\begin{array}{c}\mathrm{b}, \mathrm{b},(\mathrm{B}),(\mathrm{b}) \\
\mathrm{b},(\mathrm{B}),(\mathrm{b}),(\mathrm{M}) \\
\left(\mathrm{B}_{76}\right),\left(\mathrm{b}_{76}\right),\left(\mathrm{b}_{76}\right),\left(\mathrm{B}_{76}\right)\end{array}$ & $\begin{array}{c}\mathrm{M}, \mathrm{m},(\mathrm{b}),(\mathrm{m}) \\
\mathrm{M}, \mathrm{m}\end{array}$ & $\begin{array}{c}\mathrm{m}_{77}, \mathrm{~m}_{77}, \mathrm{M}, \mathrm{M}, \mathrm{M} \\
\mathrm{M}_{75}\end{array}$ \\
\hline
\end{tabular}

Légende : Chaque situation d'essai complet ou simplifié est représentéc dans le tableau ci-dessus par une lettre, accompagnée ou non d'indices distinctifs.

— La lettre correspond à un jugement sur l'état structural de la couche travaillée : B : Bon ; b : Moyen ; m : Médiocre ; M : Mauvais. - Les millésimes désignent les essais complets des campagnes correspondantes. Les lettres sans millésimes correspondent aux essais simplifićs de 1977.

- Les parenthèses indiquent les situations où un facteur limitant a été identifié (parasitisme-pied grave, carence en $\mathrm{K}$ ou $\mathrm{Mg}$, sécheresse en 1976).

(') Le maximum est considéré comme atteint à une dose d'azote donnéc si le nombre de grains $/ \mathrm{m}^{2}$ obtenu à cette dose est au moins égal (à « $\mathrm{n}$ » grains près) aux nombres de grains $/ \mathrm{m}^{2}$ obtenus pour les doses supérieures ; $\mathrm{n}=\frac{5 \text { quintaux/ha }}{\text { poids moyen variétal d'un grain }}$; la valeur de 5 quintaux a été choisic compte tenu de la précision de la connaissance des rendements et des
apports d'engrais.

Les indications correspondant à ces 2 principales rubriques peuvent être reportées sur le tableau 5. On obtient le tableau 7. On observe alors que :

- Les courbes de type $\alpha$ correspondent à des conditions assez variables.

- Par contre, les courbes de type $\beta$ correspondent en majorité à des situations où l'intervention d'un facteur limitant à la croissance peut être invoquée avant la floraison.

- Les courbes de type $\gamma$ ont été obtenues en grande majorité sur des parcelles à état structural défavorable.

- Les courbes de type $\delta$ ont été obtenues dans les conditions les plus favorables.

Ainsi l'étude de la réponse du nombre de grains à la fertilisation azotée amène à conserver les hypothèses précédemment émises pour expliquer les écarts observés vis-à-vis du schéma prévisionnel de la relation azote-peuplement végétal.

Au contraire, quand l'état structural de la couche travaillée est jugé convenable, la méthode convient généralement et la justesse de l'estimation de la dose dépend alors du choix de l'objectif de rendement, c'est-à-dire de la prise en compte des ressources et contraintes du milieu. Le cas de l'essai ENE en 1975, désigné par le sigle « $b_{75}$ » illustre bien l'intérêt de prendre en considération le nombre de grains $/ \mathrm{m}^{2}$ : il est classé avec ce critère en type $\alpha$, alors qu'il avait été classé en type $\beta$ d'après les rendements. Or sur cet essai, une infestation parasitaire importante n'a été observée qu'après floraison. Elle n'a donc affecté que le poids de 1000 grains. On peut considérer que si le parasitisme avait été bien contrôlé sur cet essai, le rendement à la dose $\mathrm{X}$ aurait été égal à l'objectif.

\section{Discussion sur les conditions d'application de la méthode du bilan prévisionnel}

L'interprétation des résultats obtenus conduit à conclure que la méthode des bilans peut être adoptée dans le milieu considéré dans la mesure où les conditions de validité du modèle sous-jacent sont satisfaites. Or ces conditions excluent de nombreuses situations qui ne permettent pas l'appréciation pertinente de la méthode. Il convient donc de bien les expliciter.

\section{L'azote doit être facteur limitant du rendement, de la dose nulle à la dose prévue}

Quand un autre facteur limitant, ignoré a priori, intervient, l'objectif de rendement et, par voie de conséquence, les doses d'engrais nécessaires, sont surestimés. Mais ce n'est pas la méthode elle-même qui est alors mise en défaut.

Nous avons été amenés à distinguer les facteurs limitants qui ne jouent un rôle que tardivement, durant la phase de remplissage des grains (parasitisme, déficit hydrique modéré) de ceux qui interviennent plus précocement et affectent la croissance de l'appareil végétatif et le nombre de grains à l'unité de surface. Dans le $1^{\text {er }}$ cas (exemple typique de l'essai ENE de 1975), la dose de fumure optimum n'est pas déplacée et le nombre de grains-objectif est atteint à la dose prévue. Le problème pour l'agriculteur est alors de tenter d'assurer dans la mesure du possible l'alimentation des grains formés. Nous reviendrons sur ce problème dans le $4^{\mathrm{e}}$ mémoire de cette série.

Au contraire, dans le cas d'une intervention précoce, d'autres facteurs limitants (eau en 1976, potassium, magnésium ou parasitisme du pied dans certaines parcelles en 1977), la dose de fumure optimum est, généralement, déplacée. L'agriculteur doit alors diagnostiquer et, s'il le 
peut, corriger la déficience (analyse de sol et fumure de correction, observation de fosses pédologiques et, dans certains cas, travail profond du sol pour accroître la profondeur d'enracinement). Si celle-ci n'est pas corrigible (sol à faible réserve hydrique et impropre au sous-solage, par exemple), il devra tenir compte, dans la fixation de l'objectif de rendement, d'un facteur limitant probable.

\section{Les conditions d'absorption de l'azote par le peuplement végétal sont optimales}

Ce postulat dangereusement implicite n'est satisfait que si l'absorption d'azote n'est pas perturbée par un mauvais enracinement ou par la siccité du sol. Deux cas limites se présentent :

- La couche travaillée est très desséchée à partir du début de la montaison (cas de 1976, cf. SEBILlotTE et al., 1978). Les ions, même nitriques, sont peu mobiles: les transferts de masse par écoulement (mass flow) et les flux de diffusion sont faibles. Les engrais, appliqués sur sol sec, restent hors de portée des racines fonctionnelles. La réponse du végétal à l'azote est limitée, voire totalement annulée, non parce que celui-ci n'est pas limitant, mais parce que l'azote des engrais ne peut être absorbé. Les courbes de type $\gamma$ rejoignent celles de type $\beta$.

- La couche travaillée reste constamment au voisinage de la capacité au champ (cas de 1978 dont les résultats sont analysés dans un document en préparation). En ce cas, les éléments minéraux solubles sont très mobiles et l'alimentation minérale peut être satisfaisante même en cas d'enracinement peu dense.

Entre ces 2 extrêmes se localisent les courbes de type $\gamma$ obtenues dans des circonstances climatiques telles que la couche arable se dessèche plus ou moins intensément et durablement (cas de 1975 et 1977). Les variations d'état structural de la couche travaillée ont alors une grande influence sur l'absorption d'azote, par l'intermédiaire de la densité d'enracinement et, peut-être, de la minéralisation de l'azote organique.

Ce type de circonstances climatiques intermédiaires est largement le plus fréquent dans la région considérée. Un mauvais état structural se traduit donc, pour une dose d'azote donnée, par un risque élevé de déficit de rendement.

Ainsi, en 1977, sur les situations à état structural jugé médiocre ou mauvais, on obtient en moyenne 16700 grains $/ \mathrm{m}^{2}$ à la dose $\mathrm{X}$ contre 18900 sur celles à état structural jugé moyen ou bon. Cette différence représente un déficit potentiel de rendement d'environ $8 \mathrm{q}$. Compte tenu des rapports de prix actuels et de la résistance à la verse des variétés modernes, les agriculteurs préfèrent surfertiliser et il est plus réaliste de souligner les conséquences de l'état structural sur l'économie d'azote et les risques de pollution : ce sont environ 40 unités d'azote supplémentaires qui permettent aux parcelles à profil défavorable d'obtenir un rendement équivalent à celui des parcelles qui satisfont à la prévision par bilans. Ce chiffre donne la mesure de la quantité d'azote gaspillée et disponible pour une migration en profondeur. On ne peut cependant lui accorder aucune valeur générale ni l'extrapoler tel que à d'autres conditions d'expérience.

Les causes des mauvais états structuraux, rencontrés dans environ un profil sur 2 , semblent résider principalement dans les mauvaises conditions de récolte des betteraves, dans l'emploi à mauvais escient du cover-crop substitué au labour pour ameublir le sol, enfin dans l'emploi des rouleaux pour limiter le déchaussement hivernal très redouté en terre de craie. Dans bien des cas, l'observation rapide d'un profil cultural après récolte du précédent aurait permis à l'agriculteur de mieux choisir ses techniques de travail du sol. On peut considérer l'amélioration de celles-ci comme un des progrès prioritaires à réaliser dans les exploitations de grande culture dont le calendrier de travail présente des pointes difficiles à résorber et souvent préjudiciables à la qualité des travaux au champ.

\section{Certains processus du cycle de l'azote dans le sol peuvent être négligés}

Ainsi les postes de dénitrification, fixation d'azote atmosphérique, apport par les pluies et volatilisation ne sont pas pris en compte dans le bilan prévisionnel, ce qui suppose qu'ils sont de faible ampleur et se compensent mutuellement. Cette hypothèse, non vérifiable directement dans nos conditions expérimentales, peut ne pas s'appliquer à certains milieux particuliers.

Dans les cas où les 2 conditions (1) et (2) étaient réunies, la méthode des bilans s'est avérée très satisfaisante :

- Envisagéc globalement, dans les situations où les 2 postulats étaient respectés (c'est-à-dire là où l'objectif de rendement n'était pas surestimé et où les états structural et hydrique étaient favorables), la méthode a permis dans plus de 80 p. 100 des cas d'obtenir au moins le rendement prévu à la dose prévue.

- En analysant point par point les postes du bilan qui étaient accessibles à l'observation ou au calcul, on a obtenu un assez bon accord entre les résultats expérimentaux et les normes prévisionnelles: la norme d'absorption de $3 \mathrm{~kg}$ d'azote/q de grain caractérise bien, en moyenne, les peuplements végétaux situés à l'optimum de réponse aux fumures. Les mesures de reliquats après récolte et les évaluations de minéralisation par calcul sont assez variables, mais correspondent, en moyenne, aux normes utilisées. Ainsi, en 1977, la minéralisation moyenne sur les traitements $(\mathrm{X}, \mathrm{Y})$ de 22 situations, évaluées d'après les bilans d'azote a posteriori a été de 41 unités/ha, l'estimation prévisionnelle étant de 45 unités $\left(\mathrm{N}_{\text {hum }}=20 ; \mathrm{N}_{\text {rćs }}=25\right.$ pour le précédent betterave sucrière).

\section{CONCLUSION}

L'un des principaux objets de cette étude du blé d'hiver en Champagne crayeuse auquel on s'est limité dans ce mémoire, consistait à mettre au point les recommandations relatives aux doses globales de fumure azotée.

Pour y parvenir, on a employé et testé la méthode du bilan prévisionnel, conçuc et mise au point par HÉBERT \& RÉMY dans une double perspective :

Calcul des fumures azotées et prévision des courbes de réponses que l'on a pu confronter à des données expérimentales acquises lors de 3 campagnes dans un assez grand nombre d'essais et de parcelles d'enquête. Malgré la variabilité des situations dans lesquelles la méthode a été contrôlée, elle a donné satisfaction dans la majorité des cas, dont on a vérifié a posteriori qu'ils correspondaient à son domaine de validité. On peut conseiller sa généralisation en Champagne crayeuse moyennant une adéquation précise de ses divers éléments à la variabilité spatiale du milieu; ce dernier point fera l'objet du $3^{c}$ mémoire de cette série. 
Analyse de la variabilité de la réponse du blé d'hiver à la fumure azotée, rendant compte d'une part de la variabilité de cette réponse (surtout, dans le cas étudié, celle liée à la richesse du sol en azote), le modèle prédictif permet, de surcroît, de classer les écarts prévision-réalisation. C'est l'option qui a été retenue pour élaborer une typologie des courbes de réponse. Confrontée à l'analyse du milieu et du comportement de la végétation, cette classification a permis :

- de mettre en évidence le rôle majeur de l'état structural sur l'utilisation de l'azote par le peuplement de blé. L'intérêt de cette conclusion, qui pouvait être attendue d'après les travaux expérimentaux d'autres auteurs, tient au fait qu'elle se dégage dans les conditions de la pratique agricole, avec une ampleur qui domine celle d'autres facteurs de variabilité ;

- de souligner l'importance de la prévision du rendement objectif, c'est-à-dire de la détection et du contrôle des facteurs limitants du rendement autres que l'azote. Faute d'une procédure efficace de fixation de cet objectif, la détermination des fumures par bilan prévisionnel perd toute signification pratique.

Au contraire, si une prévision précoce du nombre de grains par $\mathrm{m}^{2}$ s'avère possible (MASLE-MEYNARD, 1980) cette méthode de fixation des fumures prendra un intérêt accru, notamment pour minimiser les pertes.

D'un grand intérêt pour régulariser les rendements, accroître l'efficacité des fumures et réduire leurs effets polluants, la méthode du bilan prévisionnel doit être associée à d'autres progrès de la technicité des agriculteurs : le diagnostic réalisé a permis de mettre l'accent sur le travail du sol et la maîtrise des parasites fongiques. De plus, le rôle bénéfique de la méthode du bilan prévisionnel est indisso- ciable des modalités rationnelles d'application (stade et répartition des apports) qui ont été employées.

Globalement, sur les parcelles conduites selon ces recommandations, les rendements ont été plus élevés que chez les agriculteurs concernés, alors que les situations retenues n'étaient pas spécialement favorables. Cette appréciation positive sur l'efficacité du travail entrepris n'est pas démentie par l'évolution des rendements moyens du C.E.T.A. après diffusion des premiers résultats, manifestant une progression très sensible alors que certaines campagnes (cas de 1977) présentaient une climatologie plutôt défavorable.

Sur le plan méthodologique, il est également à noter que ce n'est pas en lui-même et à lui seul que l'outil théorique du bilan prévisionnel a été performant. La conception du dispositif d'ensemble pluriannuel et multilocal, au sein duquel a été mis en cuvre cet outil, ainsi que la pertinence des contrôles réalisés tant sur le milieu que sur le peuplement végétal, ont joué un rôle indissociable et aussi important vis-à-vis de l'obtention des conclusions. On peut même affirmer que, sans cet effort d'analyse agronomique de situations au champ, on aurait été conduit à une attitude très sceptique quant à la validité pratique de la méthode du bilan prévisionnel. L'étude des situations culturales se place à part entière dans le champ scientifique de l'agronomie : elle prolonge et enrichit l'élaboration des modèles théoriques utilisés dans cette discipline.

Reçu le 30 octobre 1980 Accepté le 25 juin 1981.

\section{REMERCIEMENTS}

Nous remercions le C.E.T.A. de Romilly-sur-Seine et son ingénicur, G. FERRATON, pour le support expérimental qu'ils nous ont offert et l'intérêt qu'ils ont porté à nos travaux.

\section{ANNEXE 1}

ANALYSE DE VARIANCE SUR LES RENDEMENTS DES ESSAIS (1975-76-77)

Les rendements exprimés en q/ha de grains à $16 \mathrm{p} .100$ d'humidité ont été calculés d'après des prélèvements effectués à la moissonneuse-batteuse. $-S=$ effet significatif au seuil $P=0,05 ; N S=$ Non significatif au même seuil.

- Les traits reliant les moyennes des traitements indiquent qu'elles sont significativement différentes (méthode du $T$ corrigé, seuil global $P=0,10)$

- Les interactions entre traitements ne sont pas significatives au seuil $p=0,05$ en 1975 et 1976.

Variance analysis of the yields obtained on the experimental plots in 1975-76-77

$S=$ significant effect at $P=0,05 ; N S:$ non significant effect at $P=0,05$

- The averages of the yields which are joined with lines are significantly different.

- The interactions between traitements are not significant at $P=0,05$ in 1975 and 1976.

\begin{tabular}{|c|c|c|c|c|c|c|c|c|c|c|c|c|}
\hline \multirow{2}{*}{1975} & \multirow{2}{*}{$\begin{array}{l}\text { Témoin } \\
\text { sans } \\
\text { azote }\end{array}$} & \multicolumn{4}{|c|}{$\begin{array}{l}\text { Moyennes du facteur } \\
\text { dose globale }\end{array}$} & \multicolumn{4}{|c|}{$\begin{array}{l}\text { Moyennes du facteur } \\
\text { dose tallage }\end{array}$} & \multirow{2}{*}{$\begin{array}{c}\text { Effet } \\
\text { bloc }\end{array}$} & \multirow{2}{*}{$\begin{array}{l}\text { Coefficient } \\
\text { de } \\
\text { variation }\end{array}$} & \multirow{2}{*}{ Remarques } \\
\hline & & $x-30$ & $\mathrm{X}$ & $x+30$ & Test & $Y-30$ & $\mathrm{Y}$ & $Y+30$ & Test & & & \\
\hline $\begin{array}{l}\text { Essai } \\
\text { Nord } \\
\text { (ENE) }\end{array}$ & 29.4 & 41.8 & 42.8 & 43.0 & NS & 42.0 & 42.1 & 43.5 & NS & S & $5.8 \%$ & \\
\hline $\begin{array}{l}\text { Essai } \\
\text { Centre } \\
\text { (ECE) }\end{array}$ & 50.1 & 59.8 & 60.7 & $\underset{ }{61.1}$ & $S$ & $\begin{array}{c}60.7 \\
\\
\end{array}$ & $\stackrel{61.7}{\longleftarrow}$ & 59.2 & $\mathrm{~S}$ & $S$ & $2.4 \%$ & $\begin{array}{c}\text { Interaction } \\
\text { bloc-dose globale } \\
\text { significative }\end{array}$ \\
\hline $\begin{array}{l}\text { Essai } \\
\text { Sud } \\
\text { (ESO) }\end{array}$ & 23.2 & $\stackrel{47.4}{\longleftarrow}$ & $\begin{array}{r}50.4 \\
\longrightarrow \\
\end{array}$ & & $S$ & 50.4 & 51.4 & 50.8 & NS & $S$ & $4.3 \%$ & \\
\hline
\end{tabular}




\begin{tabular}{|c|c|c|c|c|c|c|c|c|c|c|c|c|c|c|c|}
\hline \multirow{2}{*}{1976} & \multicolumn{2}{|c|}{$\begin{array}{l}\text { Témoin } \\
\text { sans azote }\end{array}$} & \multicolumn{3}{|c|}{$\begin{array}{l}\text { Moyennes du facteur } \\
\text { fongicide }\end{array}$} & \multicolumn{4}{|c|}{$\begin{array}{l}\text { Moyennes du facteur } \\
\text { Dose globale }\end{array}$} & \multicolumn{4}{|c|}{$\begin{array}{l}\text { Moyennes du facteur } \\
\text { Dose tallage }\end{array}$} & \multirow{2}{*}{$\begin{array}{l}\text { Effet } \\
\text { bloc }\end{array}$} & \multirow{2}{*}{$\begin{array}{c}\text { Coefficient } \\
\text { de } \\
\text { variation }\end{array}$} \\
\hline & fongic. & $\begin{array}{c}\text { sans } \\
\text { fongic. }\end{array}$ & fongic. & $\begin{array}{l}\text { sans } \\
\text { fongic. }\end{array}$ & Test & $x-40$ & $X$ & $x+40$ & Test & $Y-40$ & $\mathrm{Y}$ & $Y+40$ & Test & & \\
\hline $\begin{array}{c}\text { Essai } \\
\text { Nord } \\
\text { (ENO) }\end{array}$ & 38.6 & 33.6 & 42.7 & 40.6 & $S$ & 42.0 & 41.6 & 41.1 & NS & 41.4 & 41.3 & 42.1 & NS & $S$ & $8.8 \%$ \\
\hline $\begin{array}{l}\text { Essai } \\
\text { Sud } \\
\text { (ESU) }\end{array}$ & 46.6 & 48.1 & 52.3 & 53.2 & $S$ & 52.0 & 53.0 & 53.2 & NS & $\stackrel{51.9}{\llcorner}$ & $\stackrel{52.8}{\longrightarrow}$ & & $S$ & $S$ & $3.1 \%$ \\
\hline $\begin{array}{c}\text { Essai } \\
\text { Centre } \\
\text { Talent } \\
\text { (ECA) }\end{array}$ & 35.2 & 37.1 & 39.0 & 38.5 & NS & $\stackrel{37.9}{\longleftarrow}$ & $\begin{array}{r}39.0 \\
\\
\end{array}$ & $\begin{array}{r}39.2 \\
-\end{array}$ & $S$ & 38.6 & 38.9 & 38.7 & NS & $S$ & $3.7 \%$ \\
\hline $\begin{array}{c}\text { Essai } \\
\text { Centre } \\
\text { Top } \\
\text { (ECO) }\end{array}$ & 34.7 & 34.1 & 36.9 & 35.9 & $S$ & 36.5 & 36.0 & 37.0 & NS & 36.5 & 36.1 & 36.7 & NS & $S$ & $4.7 \%$ \\
\hline \multirow{2}{*}{1977} & \multicolumn{2}{|c|}{$\begin{array}{l}\text { Témoin } \\
\text { sans azote }\end{array}$} & \multicolumn{3}{|c|}{$\begin{array}{l}\text { Moyennes du facteur } \\
\text { fongicide }\end{array}$} & \multicolumn{4}{|c|}{$\begin{array}{l}\text { Moyennes du facteur } \\
\text { dose globale }\end{array}$} & \multicolumn{4}{|c|}{$\begin{array}{l}\text { Moyennes du facteur } \\
\text { dose tallage }\end{array}$} & \multirow{2}{*}{$\begin{array}{l}\text { Effet } \\
\text { bloc }\end{array}$} & \multirow{2}{*}{$\begin{array}{c}\text { Coefficient } \\
\text { de } \\
\text { Variation }\end{array}$} \\
\hline & fongic. & $\begin{array}{c}\text { sans } \\
\text { fongic. }\end{array}$ & fongic. & $\begin{array}{l}\text { sans } \\
\text { fongic. }\end{array}$ & Test & $X-40$ & $x$ & $x+40$ & Test & $Y-40$ & $Y$ & $Y+40$ & Test & & \\
\hline $\begin{array}{c}\text { Essai } \\
\text { Ensemble } \\
\text { Ouest }\end{array}$ & 30.8 & 28.0 & 59.8 & 50.4 & $S$ & $\stackrel{52.4}{\longleftarrow}$ & $\stackrel{55.0}{\longleftarrow}$ & $\begin{array}{r}57.8 \\
\end{array}$ & $S$ & & & & & NS & $3.2 \%$ \\
\hline $\begin{array}{c}(\mathrm{ENO}) \\
\text { traité } \\
\text { fongicide }\end{array}$ & & & & & & $\stackrel{56.1}{\longleftarrow}$ & $\stackrel{59.7}{\longleftarrow}$ & $\begin{array}{r}62.9 \\
\\
\end{array}$ & $S$ & 58.0 & 59.8 & $\begin{array}{c}61.0 \\
- \\
\end{array}$ & $S$ & $S$ & $3.5 \%$ \\
\hline $\begin{array}{c}\text { Essai } \\
\text { Est } \\
\text { Terre } \\
\text { Blanche } \\
\text { (EEB) }\end{array}$ & 37.9 & 38.5 & 66.6 & 57.8 & $S$ & $\begin{array}{l}59.0 \\
\stackrel{L}{L}\end{array}$ & 62.7 & $\begin{array}{r}64.9 \\
\longrightarrow\end{array}$ & $S$ & 62.7 & 62.4 & 61.6 & NS & NS & $4.4 \%$ \\
\hline $\begin{array}{c}\text { Essai } \\
\text { Est } \\
\text { Terre } \\
\text { Rouge } \\
\text { (EER) }\end{array}$ & 36.5 & 37.0 & 66.1 & 58.3 & $S$ & 59.4 & 63.2 & $\begin{array}{c}64.1 \\
-\end{array}$ & $S$ & 62.3 & 62.1 & 62.2 & NS & NS & $3,9 \%$ \\
\hline
\end{tabular}

\section{7 - INTERACTIONS SIGNIFICATIVES}

EON (fongicide) - Fertilisation azotée : dose globale $(D G) \times$ dose tallage $(D T)$ :

\begin{tabular}{|c|c|c|c|}
\hline D.G. & $Y-40$ & $\mathrm{Y}$ & $Y+40$ \\
\hline$x-40$ & 56.3 & 56.5 & 55.6 \\
\hline$X$ & 58.4 & 59.0 & 61.6 \\
\hline$x+40$ & 59.3 & 63.8 & 65.6 \\
\hline
\end{tabular}

$E E B$ - traitement fongicide $\times$ dose globale d'azote :

\begin{tabular}{|c|c|c|c|}
\hline D.G. & $x-40$ & $X$ & $x+40$ \\
\hline Traité fongicide & 61.9 & 67.2 & 70.7 \\
\hline Non traité & 56.1 & 58.2 & 59.1 \\
\hline
\end{tabular}

$E E R$ - traitement fongicide $\times$ dose globale d'azote

\begin{tabular}{rccc}
\hline \hline D.G. & $X-40$ & $X$ & $X+40$ \\
\hline traité fongicide & 62.2 & 67.0 & 69.3 \\
\hline non traité & 56.5 & 59.4 & 59.0 \\
\hline
\end{tabular}




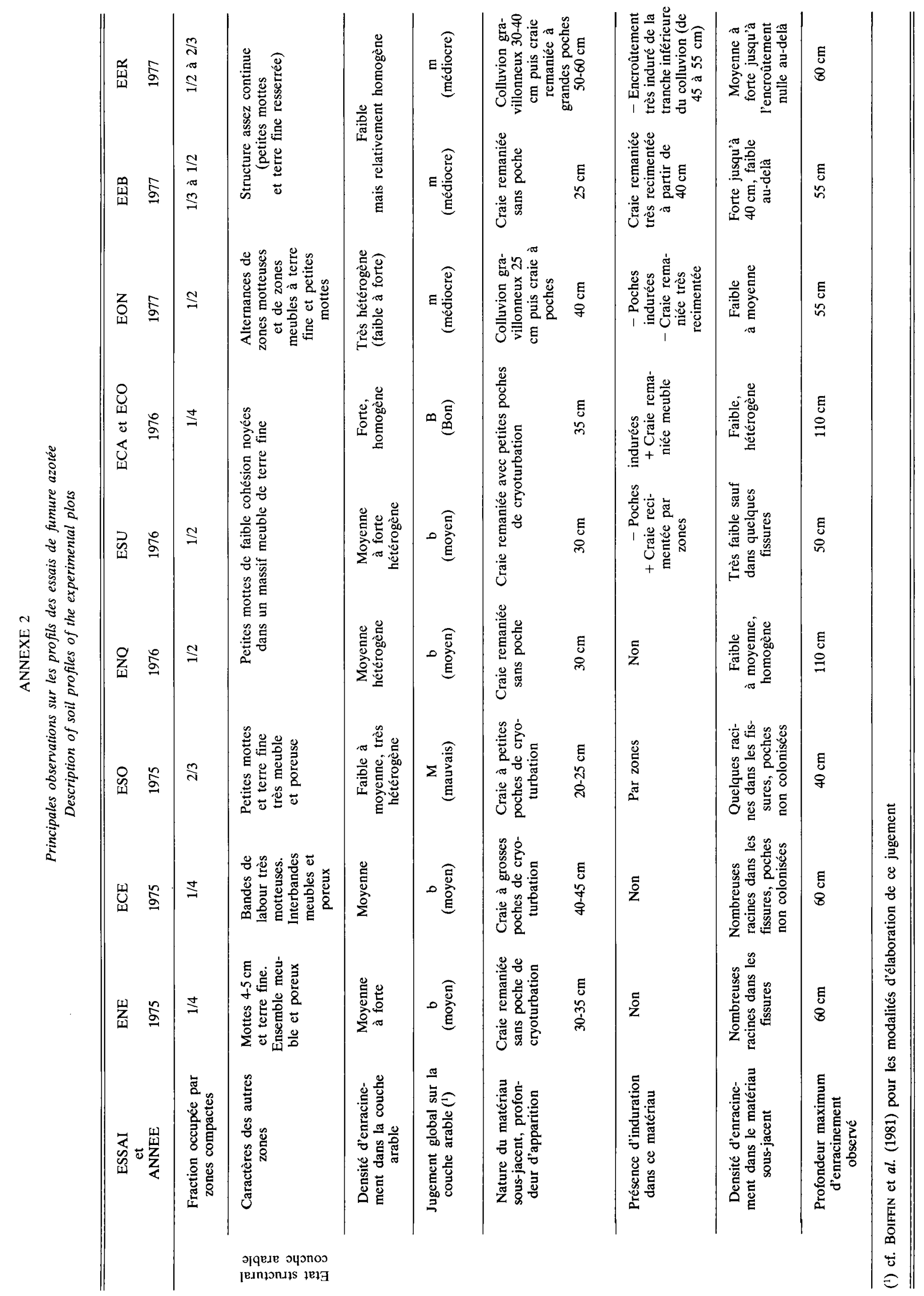




\section{RÉFÉRENCES BIBLIOGRAPHIQUES}

Blanchet R., Maertens C., Bosc M., 1974. Principaux factcurs agronomiques déterminant l'absorption des éléments nutritifs par les racines. Sci. Sol, 69-79.

Boiffin J., Caneill J., Meynard J. M., Sebillotte M., 1981. Elaboration du rendement et fertilisation azotée du blé d'hiver en Champagne crayeuse. I - Protocole et méthode d'étude d'un problème technique régional. Agronomie, 1 (7), 549-558.

Coic Y., 1956. La nutrition et la fertilisation du blé d'hiver. Les besoins en azote du blé d'hiver. Conséquences agronomiques. Ann. agron., 7 (1), 115-131.

Draycott A. P., Hull R., Messem A. B., Webb D. J., 1970. Effect of soil compaction on yield and fertilizer requirement of sugar beet. $J$. agric. Sci. Cambridge, 75, 533-537.

Ferraton G., 1975, 1976, 1977. Comptes rendus de réunion du C.E.T.A. de Romilly-sur-Seine (Aube). Nos 203, 211, 219 (32, 47 et $36 \mathrm{pp}$.).
Hébert J., 1969. La fumure azotée du blé tendre d'hiver. Bull. techn. Inform. Min. Agric., 224, 755-766.

Hébert J., 1975. Données récentes sur la fertilisation du blé in Techniques nouvelles de production du blé, I.T.C.F., 142-195.

I.T.C.F., 1975. Adaptation régionale d'une méthode de prévision de la fumure azotée du blé d'hiver (essais $17 \mathrm{NP}$ ). Ronéo. $22 \mathrm{pp}$.

Masle-Meynard J., 1980. L'élaboration du nombre d'épis chez le blé d'hiver. Influence de différentes caractéristiques de la structure du peuplement sur l'utilisation de l'azote et de la lumière. Thèse de Docteur-Ingénieur. I.N.A.-P.G. Paris, 201 pp. + annexes.

Rémy J. C., Hébert J., 1977. Le devenir des engrais azotés dans le sol. C.R. Acad. Agric. Fr., 63 (11), 700-710.

Sébillotte M., Boiffin J., Caneill J., Meynard J. M., 1978. Sécheresse et fertilisation azotée du blé d'hiver. Essai d'analyse de situations au champ par l'étude des composantes du rendement. Bull. Assoc. Fr. Etud. Sol, 3, 197-214. 